\title{
Orbital medial wall fractures: purely endoscopic endonasal repair with polyethylene implants
}

\author{
Colletti, G., * Pipolo, C., ${ }^{\dagger}$ Lozza, P., ${ }^{\dagger}$ Felisati, G., ${ }^{\dagger}$ Allevi, F., ${ }^{\dagger}$ Biglioli, F., ${ }^{\dagger}$ Deganello, A. ${ }^{\star}$ \& Saibene, A.M. ${ }^{\dagger}$ \\ ${ }^{*}$ Maxillofacial surgery department, ${ }^{\dagger}$ Otolaryngology department, San Paolo Hospital, Università degli Studi di Milano, Milan, \\ \$Otolaryngology department, Careggi Hospital, University of Florence, Florence, Italy \\ Accepted for publication 11 May 2016 \\ Clin. Otolaryngol. 2016, 00, 00-00
}

\section{Introduction}

The lamina papyracea of the ethmoid bone owes its name to its paper-thin papyrus-like thickness, which makes fractures extremely common. According to some case series, medial wall fracture is the most frequent among orbital fractures. ${ }^{1}$ Small, isolated blow-out medial wall fractures generally may not require treatment, but early or late enophthalmos might become an aesthetic concern for patients. This complication is very likely to occur with fractures exceeding $1 \mathrm{~cm}^{2}$ or $50 \%$ of the wall. ${ }^{2}$ Furthermore, patients may also suffer from late functional problems, due to fracture site scarring which impairs eye movement.

Transfacial approaches still retain their value today, ${ }^{3}$ while several anecdotal transnasal microscopic and endoscopic approaches have been proposed since the early 90 . These approaches not only differ in terms of approach, but also for the materials employed for the reconstruction.

Recently, some small case series shed a new light on the soundness of endoscopic approaches. ${ }^{4}$ Most authors rely on the use of Silastic sheets and nasal packing to maintain the orbital content in place, with obvious drawbacks. ${ }^{2}$ Use of resorbable panels or autologous materials (bony fragments or septal cartilage) has been proposed in case reports only. Non-resorbable materials, such as titanium meshes, have been traditionally limited to transfacial approaches, ${ }^{5}$ implementing also computer-aided design for better aesthetic results. ${ }^{3}$

Given the superior stability given by non-resorbable implants and the chances provided by modern biocompatible materials, we introduced a new purely endoscopic

Correspondence: G. Colletti, Maxillofacial surgery department, San Paolo Hospital, Università degli Studi di Milano, Piazza della Repubblica 21, 20124, Milan, Italy. Tel: +39-3334312800 Fax: +39 0250323166 e-mail: giacomo.colletti@gmail.com

The work was accomplished in the San Paolo Hospital in Milan; all authors were working in their respective department, that is Colletti, Biglioli and Allevi in the maxillofacial surgery department and Pipolo, Lozza, Felisati and Saibene in the otolaryngology department. Dr. Deganello acted as otolaryngologist consultant for his experience in orbital surgery. technique employing a porous polyethylene implant (Medpor, Stryker, Kalamazoo, MI, USA). Our technique couples the stronger support granted by non-resorbable materials and the minimal invasiveness of the endoscopic approach without the need for long-term nasal packing.

\section{Technical description}

All our patients undergo a head and neck examination, an ophthalmologic evaluation and a plain CT scan to evaluate indications and plan the approach.

The procedure is undertaken under general anaesthesia within 5 days after the trauma.

The patient is placed in the standard ESS surgical position, leaving both eyes uncovered. The procedure is performed using a rigid 4-mm endoscope, usually a $0^{\circ}$ scope. Angled scopes can be used, according to the surgeon's preferences. With endoscopic cutting forceps, a standard radical anteroposterior ethmoidectomy is performed on the affected side, carefully approaching the herniated orbital content. The fractured lamina papyracea is then completely removed freeing the intact part surrounding the fracture. If needed, bipolar cautery is selectively applied to any visible source of bleeding. Before commencing the reduction of the soft tissues, two prerequisites must be fulfilled: no spare fractured bone must be left in the soft tissues to prevent it to impinge the medial rectus and all the four healthy margins of the fracture must be clearly identified (Fig. 1). A plain 0.8-mm Medpor sheet is cut and modelled in a convex shape roughly mimicking the desired periorbital curvature. The Medpor sheet is then placed in the nasal cavity and gently pushed beyond the non-fractured margins of the papyracea (Fig. 2). The implant shape and curvature can be modified so as to perfectly match the wall to be reconstructed. During the reconstructive procedure, we find particularly useful to adopt a $45^{\circ}$ scope. The middle meatus is packed with absorbable porcine gelatin sheets (Spongostan special, Ethicon, Johnson \& Johnson, Edinburgh, UK), and a single nasal pack (Merocel, Medtronic, Minneapolis, MI, USA) 


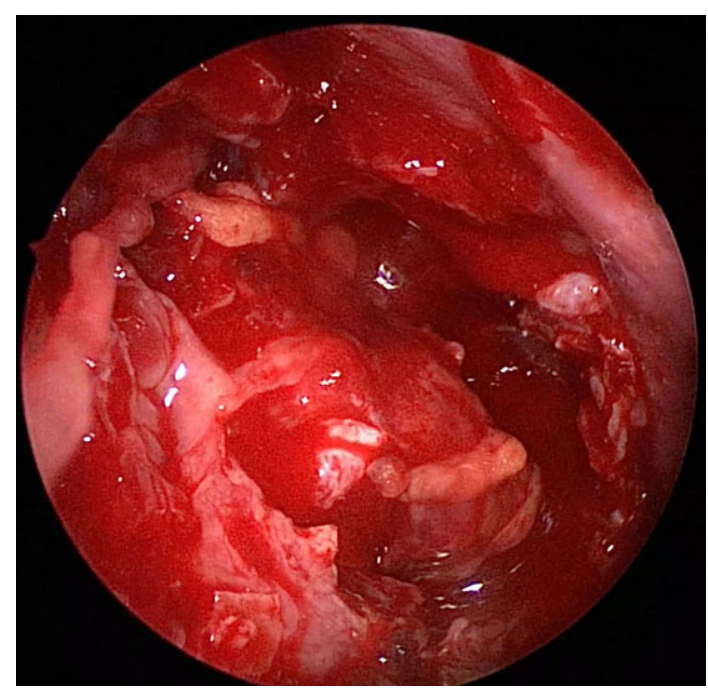

Fig. 1. Intra-operative endoscopic image showing the complete removal of the fractured lamina papyracea. The fatty content of the orbit is free to bulge inside the nasal cavity. The intact part of the lamina papyracea remains in place.

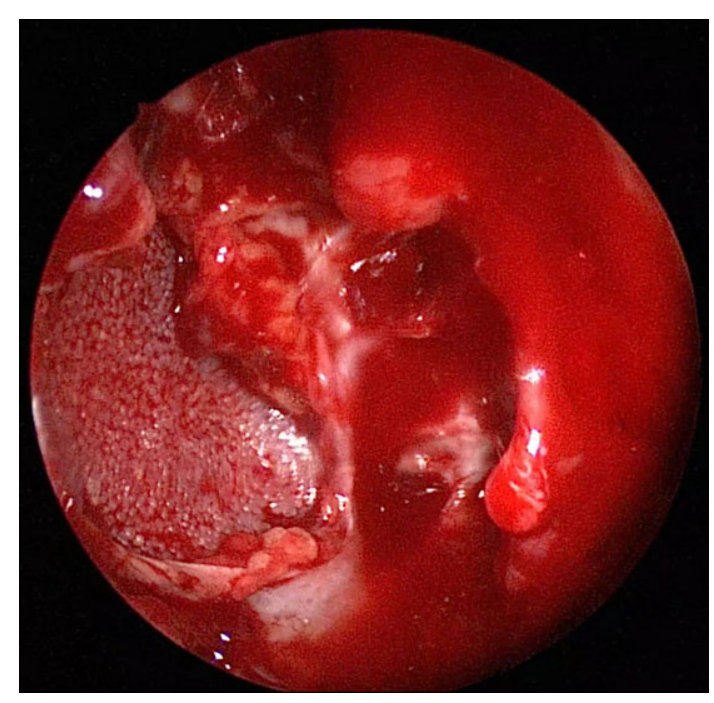

Fig. 2. Intra-operative endoscopic image showing the Medpor sheet in place after the reconstruction is complete. The Medpor sheet is positioned as an underlay of the intact portion of the lamina papyracea.

covered in a glove finger is used to avoid any nasal bleeding in the early postoperative period.

The patient is administered $1 \mathrm{~g}$ i.v. cefazolin intraoperatively and t.i.d. $1 \mathrm{~g}$ oral amoxicillin clavulanate for 5 days postoperatively.

The nasal pack is removed the day following the procedure, and the patient is dismissed on the second day after a CT scan confirming the desired correction has been achieved and an ophthalmologic evaluation ruled out any visual damage.
The patients are instructed to perform nasal douches with saline and to apply a nasal mupirocin ointment 4 times a day until the final ENT evaluation 1 month later. Until then, patients are advised to sneeze with their mouth open and avoid blowing their nose.

In our preliminary experience in six patients (with a 6- to 12-month follow-up), no complications were reported. No implant extrusion was reported, and all results were stable. The operative time ranged from 30 to 60 min (mean $40 \mathrm{~min}$ ) with a shallow learning curve, the only novelties being modelling and stabilizing the implant.

\section{Discussion}

For years, the standard approach for medial wall fractures involved an external transcutaneous 'Lynch' incision or, preferably, a transconjunctival with retrocaruncular approach, the latter still being considered as the gold standard.Some recent papers focused on endoscopic management of medial wall fractures. The first one is a case series published by Gerbino and colleagues ${ }^{5}$ : they compared the results achieved through an external approach or with a transnasal endoscopic check of the procedure. Interestingly, the authors verified that the most predictable results were those obtained with the endoscopic check, with a better control of the posterior and the upper margin of the fracture.

Other authors have proposed their case series of transnasal management of medial wall fractures with (Copelli and colleagues ${ }^{6}$ ) or without (Pagnoni and colleagues ${ }^{4}$ ) the aid of navigation. These authors, however, did not reconstruct the bony wall and relied upon long-term nasal packing (2-3 weeks) to obtain the desired correction. It seemed straightforward for us to conjoin the ideal control of the fracture, allowed by the endoscopic approach, with the reconstruction of the fractured wall with alloplastic sheets, which are routinely employed in transfacial approaches. Concerns about the safety of alloplastic materials have been raised, but new biocompatible materials such as Medpor provide stable, resistant surfaces which are less prone to host microorganisms growth than conventional metal meshes.

Using these materials, the purely endoscopic endonasal allows a better outcome than transfacial approaches from a technical and aesthetic point of view. In contrast with transfacial approaches, the lack of cutaneous incision, the lower patient discomfort and the better exposition of the surgical field with negligible muscular damage risk must be taken into account. The only drawback could be the skill required for performing an endoscopic endonasal orbital access, which should in our opinion be part of the cultural background of any rhinologist. 


\section{Acknowledgements}

None.

\section{Conflict of interest}

The authors have no conflict of interests pertaining this work. They have no financial disclosures to make.

\section{Funding}

None.

\section{Keypoints}

- Orbital medial wall is the most common among orbital fractures.

- Endoscopic endonasal repair of orbital medial wall fractures grants minimal invasivity and better control of posterior and superior fracture margins.

- New non-absorbable biocompatible materials can be employed also endoscopically, allowing for durable reconstructions without infective risks.

\section{References}

1 Choi K.E., Lee J., Lee H. et al. (2015) The paradoxical predominance of medial wall injuries in blowout fracture. J. Craniofac. Surg. 26, 752-755

2 Burnstine M.A. (2002) Clinical recommendations for repair of isolated orbital floor fractures: an evidence-based analysis. Ophthalmology 109, 1207-1213

3 Kang S.J. \& Kim J.W. (2012) Surgical treatment of enophthalmos using an endoscope and T-shaped porous polyethylene fabricated with a mirror image. Int. J. Oral Maxillofac. Surg. 41, 1186-1191

4 Pagnoni M., Giovannetti F., Amodeo G. et al. (2015) Endoscopic endonasal versus transfacial approach for blowout fractures of the medial orbital wall. J. Craniofac. Surg. 26, 247-249

5 Gerbino G., Zavattero E., Viterbo S. et al. (2015) Treatment of orbital medial wall fractures with titanium mesh plates using retrocaruncular approach: outcomes with different techniques. Craniomaxillofac. Trauma Reconstr. 8, 326-333

6 Copelli C., Manfuso A., d'Ecclesia A. et al. (2015) Endoscopic transnasal approach and intraoperative navigation for the treatment of isolated blowout fractures of the medial orbital wall. J. Craniomaxillofac. Surg. 43, 1974-1978 